\title{
Two Improved Methods for Testing ADC Parametric Faults by Digital Input Signals
}

\author{
Xiaoqin Sheng and Hans G. Kerkhoff \\ Testable Design and Testing of Integrated Systems Group, CTIT, University of Twente, Enschede, the Netherlands
}

\section{INTRODUCTION}

$\mathrm{N}_{\mathrm{p}}$ OWADAYS, mixed-signal testing becomes a challenge in production test. More and more analogue-to-digital converters (ADC) are integrated into platform-based designs. These designs are usually for video, audio and high-speed communication systems. As the systems develop, the speed and resolution of the ADCs in the platform designs become higher accordingly. As a result, higher quality analogue signal sources are required in the conventional production test, which leads to an increase of the test cost. [1] One of the most efficient ways to reduce the production test cost is multi-site testing, which can test a number of devices under test (DUT) in parallel. However, increasing the number of ADCs under test usually requires more high-quality analogue signal sources, which are very expensive [2]. In this case, it weakens the advantage of multi-site testing. Compared to an analogue signal, a digital signal is much easier and inexpensive to generate in a platform-based design, which is composed of memories, RF and mixed-signal front ends and importantly the multiple-processor cores in general. In [3], an adapted digital waveform is explored to test an ADC. However, the spectrum of a pulse wave is distorted with harmonics related to the pulse rise and fall times and duty cycle, making accurate determination of ADC parametric faults complex and time consuming [3]. In this paper, we propose two methods to improve the results in [3]. One method explores using a multiple-level digital stimulus to test the ADC. The other method applies an improved postprocessing method. Both of the methods are expected to be a quick pre-test for discarding the faulty devices by a signature result. After that, only the fault-free devices are tested by the complex conventional test. In this way, test time and cost can be saved.

In the past years, some published works have dealt with decreasing the cost and requirement of the accurate analogue input stimulus for ADC testing. In the work [4-6], the authors used two low-resolution ramp signals with offset to test the high-resolution ADC. By applying a stimulus error identification and removal algorithm (SEIR), a 16-bit ADC can be accurately tested by a 7-bit ramp signal. The authors in [7] generated a stair-case signal by a pulse-width modulation (PWM) signal and an off-chip low-pass filter. As the staircase signal is taken as the test stimulus, the $3^{\text {rd }}$ harmonic distortion of an ADC up to twenty bits can be tested with a $3^{\text {rd }}$ order polynomial fitting algorithm. The test accuracy is primarily limited by the linearity of the low-pass filter. The reference [8] presents a work that using a fully binary PWM input signal simultaneously tests the gain, offset, $2^{\text {nd }}$ and $3^{\text {rd }}$ harmonics and signal-to-noise-ratio of an ADC. It is only suitable for $\sum \Delta$ ADCs. A white noise is employed as the test stimulus in [9]. In the spectrum of the white noise, all the frequencies are expected to equally appear. As the nonlinearity of ADCs will introduce some extra frequencies, the non-linear faults of ADCs can be detected by analyzing the extra frequencies at the ADC output.

\section{ADC Testing Using A Multi-Level Pulse Wave INPUT SIGNAL}

An adapted pulse wave can be expressed in the time domain as:

$$
\begin{aligned}
& x(t)=\frac{A}{T_{r}} t\left(u(t)-u\left(t-T_{r}\right)\right) \\
& +A\left(u\left(t-T_{r}\right)-u\left(t-\left(T_{r}+T_{h}\right)\right)\right) \\
& \left.+A\left(1+\frac{T_{h}+T_{r}-t}{T_{f}}\right)\left(u\left(t-\left(T_{r}+T_{h}\right)\right)-u\left(t-T_{r}+T_{h}+T_{f}\right)\right)\right)
\end{aligned}
$$

, where $A, T_{r}, T_{f}$ and $T_{h}$ denote the amplitude, rise and fall time of the signal and time of high-level, respectively. According to the Poisson formula, discrete time derivative is applied to the time-domain function in (1). As a result, the spectrum of the adapted pulse wave can be found as:

$$
\begin{aligned}
& F\{x(t)\}=\frac{A}{\omega^{2} T_{r}}\left(e^{-j \omega T_{r}}-1\right)-\frac{A}{\omega^{2} T_{f}}\left(e^{-j \omega T_{h}}-1\right) e^{-j \omega\left(T_{r}+T_{h}\right)} \\
& -\frac{2 j A}{\omega^{2} T_{r}} \sin \omega \frac{T_{r}}{2} e^{-j \omega \frac{T_{r}}{2}}+\frac{2 j A}{\omega^{2} T_{f}} \sin \omega \frac{T_{f}}{2} e^{-j \omega\left(T_{r}+T_{h}+\frac{T_{f}}{2}\right)}
\end{aligned}
$$

Spectral representation of the adapted pulse wave in (2) shows that compared to the ideal spectrum of a pure sine wave only containing the fundamental frequency, it also contains other harmonics depending on the rise and fall times and the duty cycle. In [3], an ADC is evaluated through the FFT analysis, using an adapted pulse wave as the test stimulus. The rise or fall time of the pulse wave stimulus should be set properly to hit all the output codes of the ADC under test. Based on the requirement of Nyquist limits and the principle of coherent sampling, the rise/fall time should be at least larger than the reciprocal of the sampling frequency of the ADC under test $1 / f_{s}$. The approximate values of the dynamic parameters of the ADC can be obtained. In the simulation, to obtain the fault-free range, Monte Carlo simulations are carried out on the fault-free ADC by the pulse wave stimulus. As a result, a decision of pass or fail is made by the approximate values to distinguish the faulty devices from the fault-free devices. In real test, a number of golden 
devices, which are fault-free devices tested by the conventional method, are tested by the pulse wave to obtain the fault-free range. The number of the golden device should be sufficiently large to cover the process variations. However, the results obtained in [3] can not fully cover all types of parametric faults. It is caused by the difference of the spectrum between sine and pulse waves.

One of the potential methods to improve the result is approximating to the spectrum of a sine wave by adjusting the shape of a digital input stimulus. A sine wave can be considered as a stair-case pulse wave with countless voltage levels. The more voltage levels in a stair-case pulse wave, the more the spectrum of the pulse wave will be similar to the one of a sine wave. Nowadays, most of the ADCs are integrated into a platform-based design. In general, there are multiple power supply levels (usually three levels in maximum) in a platform design. In this case, it provides the potential to generate a pulse wave with different levels. Compare with generating an accurate analogue sine wave onchip, a stair-case pulse wave is easier and less expensive to generate in such a kind platform-based design. As a starting point, the stair-case pulse wave with multi-level in Fig.1. is applied to both golden devices and the DUTs as the test stimulus. The output spectra can be obtained respectively via FFT analysis. Compared to a single-level pulse wave input stimulus, the output spectrum of the multi-level pulse wave is more close to the sine wave. In this case, one can expect that the output spectrum from a multi-level pulse wave test stimulus can detect the parametric faults in a more sensitive way as compared to its binary counterpart. In [7], a stair-case waveform is also applied for testing the $3^{\text {rd }}$ harmonic distortion of an ADC. The method is based on the polynomial fitting technique and requires high linearity of the rise/ fall slopes of the stair-case waveform.

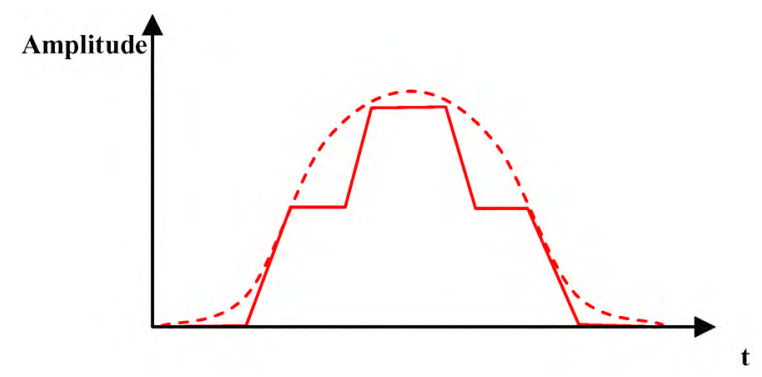

Fig. 1. The multi-level pulse wave for ADC testing

\section{ADC TESTING BY COMPARING THE DEVIATION OF THE OUTPUT SPECTRUM}

In [3], we only use the conventional way to evaluate the output spectrum, like calculating the total harmonic distortion (THD). However, the complexity of the spectrum of a pulse wave and the nonlinearity of the ADC makes the variation of the output spectrum very different from the one obtained from a sine wave stimulus. In the second method, we focus on the evaluation method of the test output and still keep using a pulse wave as the test input signal. Instead of calculating the conventional dynamic parameters, we only calculate the similarity of the output spectrums between the golden devices and the DUTs. As shown in Fig. 2, a singlelevel pulse is applied to both golden devices and the DUTs as the test stimulus. After the FFT analysis, the output spectra of golden devices and the DUTs can be obtained respectively. After that, a correlation algorithm is used to calculate the similarity between the output spectrums of the golden devices and the DUTs. If the result shows their spectra are not sufficiently similar, the device is considered to be a faulty device. Otherwise, it is fault-free. In [10], a specific correlation is used to evaluate the output results obtained from a pulse wave as well. However, the correlation is applied in the time domain of the output waveform while we apply the correlation in the frequency domain of the output in this paper. The out-of-range-percentage (OFRP) is used to distinguish faulty devices from fault-free devices.

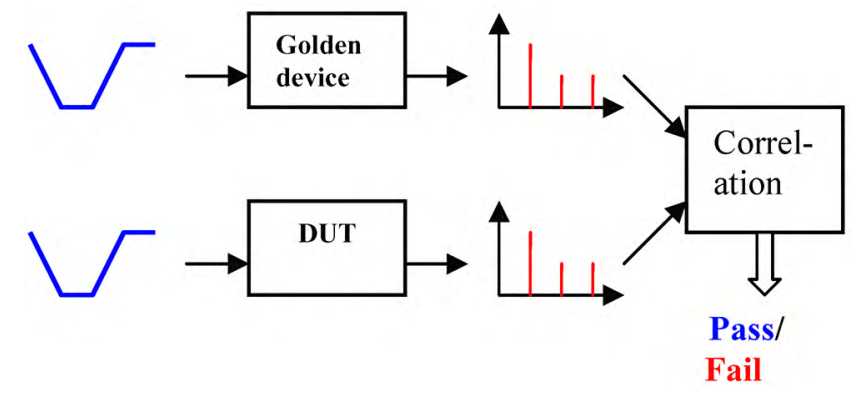

Fig. 2. ADC testing by applying the correlation between output spectrums

\section{THE DEVICE UNDER TeSt AND FAULT INJECTION}

Both of the previous improved methods are evaluated at the transistor-level design of a 6-bit flash ADC as shown in Fig. 3. Flash ADC is the fastest and simplest converter so far. An N-bit flash converter contains $2^{\mathrm{N}-1}$ comparators. The analogue input is connected to one side of the comparators while the other side of the comparators is connected to reference voltages. These reference voltages represent $2^{\mathrm{N}-1}$ voltage levels between zero and full scale. Because of the large number of comparators, several detrimental effects have to be concerned: large die size, high power dissipation, complicated clock and input signal distribution, heavy loading of the clock driving circuits [11]. As a result, it is difficult to implement a flash ADC above eight bits, especially when the low power dissipation is required.

In this work, we only focus on the parametric faults in the analogue part of the ADC. As shown in Fig. 3, the original input is applied to the first stage. The following stages will amplify and divide the input into several resolution levels through the resistor ladders. In the meanwhile, any fault in the first stage will be amplified by the following stages too. As a result, the ADC is most sensitive to the faults in the first 
stage. To evaluate the improved test methods, parametric faults are injected into the first stage which is composed of 11 pre-amplifiers. The amplifier is randomly chosen to inject three types of faults:

1) offset fault - insert a dc voltage source into the gate of the one of the input pair transistors

2) gain fault - change the resistance of the load resistors of the amplifier

3) bandwidth fault - insert an extra capacitor at the output of the amplifier

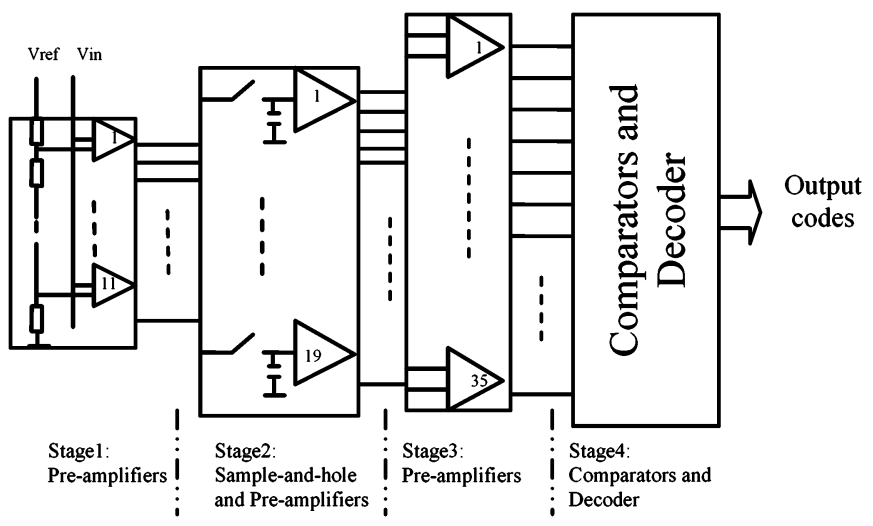

Fig. 3. The block level diagram of the 6-bit flash ADC

\section{Simulation RESUlts}

\section{A. ADC testing using multiple-level pulse wave input signal}

Three pulse waves with a different number of voltage levels are applied to the ADC respectively. As shown in Fig. 4 , all the pulse waves have the same the frequency $f_{i n}=7 \mathrm{MHz}$, the total rise/fall time $T_{r} / T_{f}=30 \mathrm{~ns}$, the lowest voltage level $V_{\text {low }}=-0.46 \mathrm{~V}$ and the highest voltage level $V_{\text {high }}=0.46 \mathrm{~V}$. The sampling frequency of the ADC is $300 \mathrm{MHz}$.

The results of the THD values with offset faults of the first-stage pre-amplifier are shown in Fig. 5. The x-axis indicates the ratio between the faulty offset and the fault-free offset, while the y-axis denotes the THD values of the ADC output obtained by the input pulse waves. The three curves in different colours represent the results by those three different types of pulse waves respectively. One can see that as the number of voltage levels increases the change of the THD value by the offset faults is more obvious. It means that the THD results can reflect the faults in a more sensitive way.

After running the simulation with all the three types of faults respectively, the results by different types of input stimuli are shown in Table 1 . In each type of fault, 8 different faulty cases are injected in total. The number of faulty cases, which are detected by the input stimulus, is listed in Table 1 . It shows that with the gain and bandwidth faults, the sine wave input stimulus can detect the faults better than all the other pulse waves input stimuli. However, with the offset faults, the 3-level pulse wave input signal can detect the fault as well as the sine wave input signal. The 4-level pulse wave is even more sensitive to the offset fault than the sine wave. It can detect 6 faulty cases while the sine wave can only detect 5 faulty cases.

With the gain and bandwidth fault, the sine wave input stimulus can detect the faults much better than the pulse wave. However, with the offset faults, the multi-level pulse wave can detect the faults even better than the sine wave input signal.

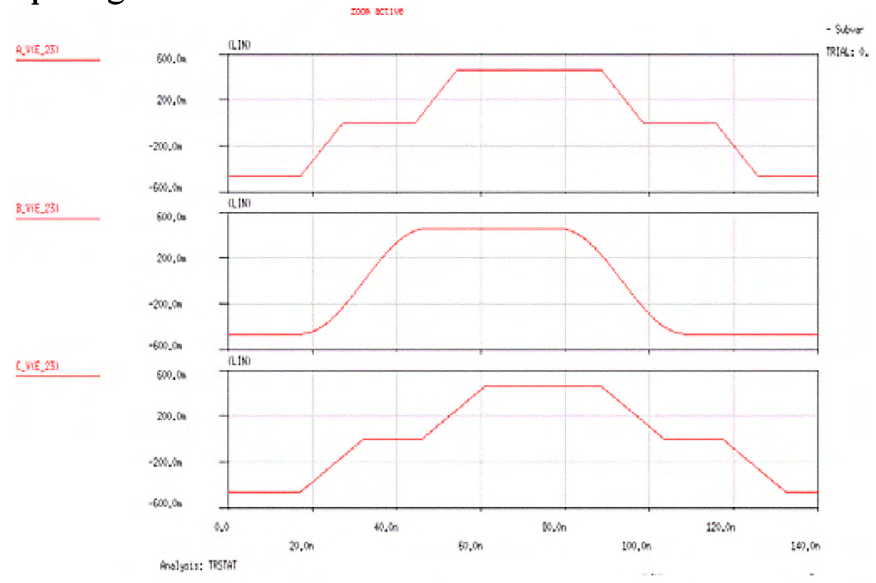

Fig. 4. The input pulse waves with different levels

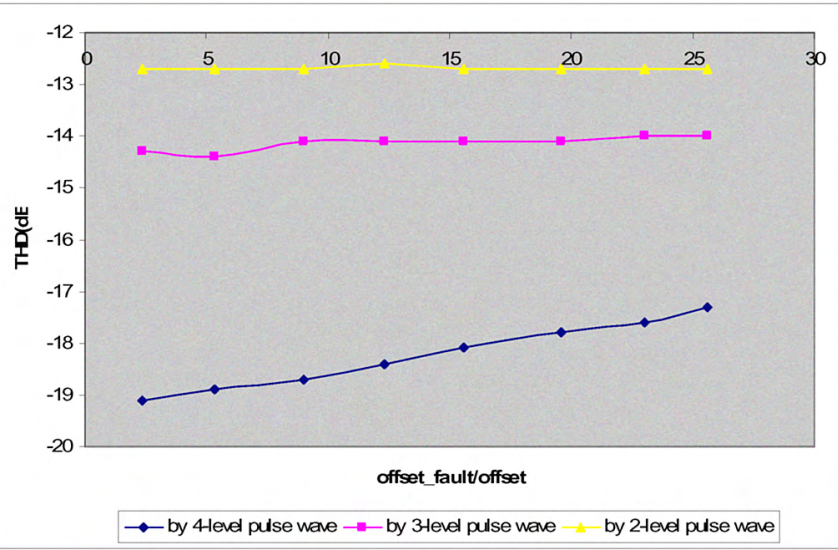

Fig. 5. The results of the THD by pulse waves input signals

TABLE I

THE NUMBER OF DETECTED FAULTY CASES BY DIFFERENT TYPES OF INPUT STIMULI

\begin{tabular}{|c|c|c|c|c|}
\hline Input stimulus & $\begin{array}{c}\text { 2-level } \\
\text { pulse } \\
\text { wave }\end{array}$ & $\begin{array}{c}\text { 3-level } \\
\text { pulse } \\
\text { wave }\end{array}$ & $\begin{array}{c}\text { 4-level } \\
\text { pulse } \\
\text { wave }\end{array}$ & $\begin{array}{c}\text { Sine } \\
\text { wave }\end{array}$ \\
\hline Offset faults & 0 & 5 & 6 & 5 \\
Gain faults & 0 & 0 & 0 & 6 \\
Bandwidth faults & 0 & 0 & 0 & 8 \\
\hline
\end{tabular}

B. ADC testing by comparing the deviation of the output spectrum

In practice, the input pulse wave has the jitter and variation of the rise/fall time. In order to see these effects to the output 
results, three different types of input stimuli are applied to the ADC:

Ideal pulse wave: $V_{\text {high }}=0.46 \mathrm{~V}, V_{\text {low }}=-0.46 \mathrm{~V}, T_{r}, / T_{f}=10 \mathrm{~ns}$, $f_{\text {in }}=7 \mathrm{MHz}$

Pulse wave with jitter: add a random jitter into the ideal pulse wave, spectral frequency density $=2.67 * 10^{-3}$, bandwidth $=$ $3.1 \mathrm{GHz}$.

Pulse wave with jitter and rise/fall time change: make the rise/fall time $0.1 \mathrm{~ns}$ different from the pulse wave with jitter.

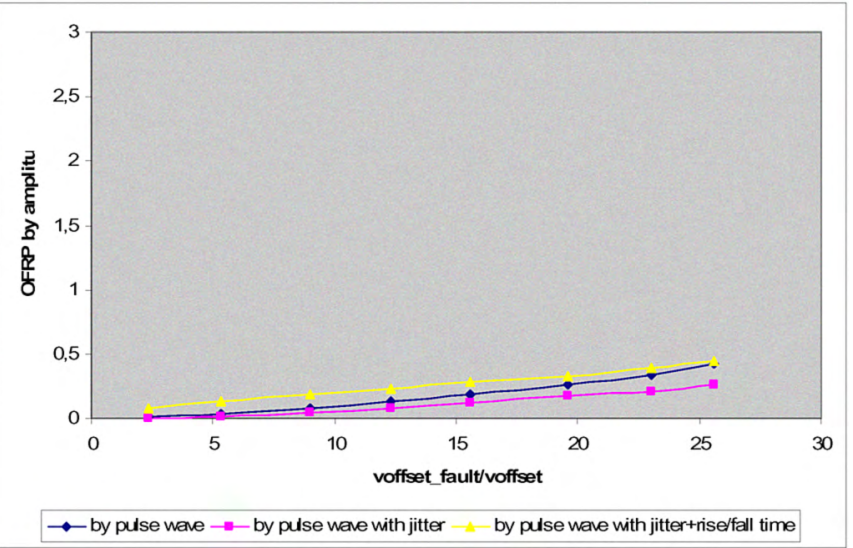

Fig. 6. The OFRP with offset faults by comparing the output amplitude

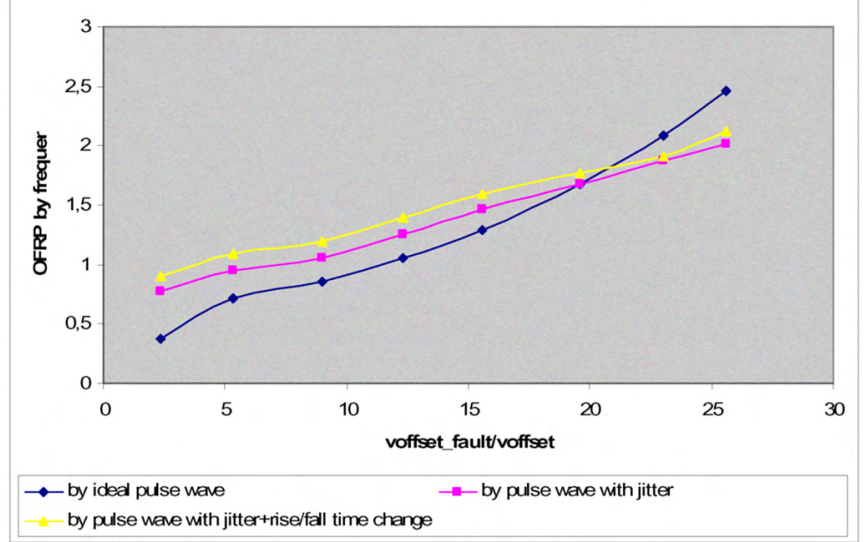

Fig. 7: The OFRP with offset faults by comparing the output spectrum

Fig. 6 shows the results of the OFRP with the offset faults by comparing the deviation of the output amplitude. The $\mathrm{x}$ axis denotes the ratio between the faulty offset and the faultfree offset, while the y-axis denotes the values of the OFRP. The OFRP results with the same offset faults by comparing the deviation of the output spectrum are shown in Fig. 7. The denotation of the x-axis and y-axis is the same as in Fig. 6 . The three curves in different colours represent the results by three different input stimuli respectively. One can observe that the values of the OFRP are affected by jitter and rise/fall edge variation in both methods. In Fig. 6 , the maximum difference between the $O F R P$ tested by the ideal pulse wave and pulse wave with jitter and rise/fall time change is about 0.25 . In Fig. 7, the maximum difference is around 0.5 . However, the $O F R P$ is much more sensitive to the faulty cases by comparing the deviation of the output spectrum. With the same faulty cases, the range of the deviation of the $O F R P$ is almost 4 times in Fig. 7 larger than the one in Fig. 6. In this case, with the offset faults, the OFRP tested by comparing the output spectrum is less sensitive to the jitter and rise/fall edge variation of the input pulse wave compared with the OFRP tested by comparing the output amplitude.

For the other two types of faults, the results of the OFRP are similar to the ones with offset faults that are less affected by the jitter and rise/fall edge variation when comparing the output spectrum.

In this method, a signature result can be obtained by the parameter $O F R P$. With all the three types of faults, it is more sensitive to the faults and less sensitive to the jitter and rise/fall time change than the previous method [10].

\section{CONCLUSION}

In this paper, two improved methods are presented extending our previous work. The first one improves the results by adjusting the voltage levels of the input pulse wave stimulus. Compared with the sine wave input stimulus, the four-level pulse wave can detect even more faulty cases with the offset faults. The second one improves the results by calculating the similarity of the output spectra between the golden devices and the DUTs. Compared with the previous method [10], it is less sensitive to the jitter and the change of the rise/fall time of the input pulse wave stimulus. In these two methods, a number of golden devices are tested at first to obtain the fault-free range. At last, a signature result is obtained from both methods. It can filter out the faulty devices in a quick way before testing the specific values of the conventional dynamic and static parameters.

\section{REFERENCES}

[1] M. Burns, G.W. Roberts, An Introduction to Mixed-Signal IC Test and Measurement, Oxford University Press, 2000.

[2] C. Wegener, M. P. Kennedy, "Innovation to Overcome Limitations of Test Equipment", Circuit Theory and Design Conference, pp. 309-314, 2005.

[3] Xiaoqin Sheng, Hans Kerkhoff, Amir Zjajo, Guido Gronthoud, "Exploring dynamics of embedded ADC through adapted digital input stimuli", IEEE Mixed-Signals, Sensors, and Systems Test Workshop, pp. 130-136, 2008

[4] L. Jin, K. Parthasarathy, T. Kuyel, "Linearity Testing of Precision Analog-to-Digital Converters Using Stationary Nonlinearity Inputs", International Test Conference, pp. 218-227, 2003.

[5] L. Jin, C. He, D. Chen, R. Geiger, "Fast Implementation of a Linearity Test Approach for High Resolution ADCs Using Non-linear Ramp Signal", International Symposium on Circuits and Systems, pp. 932-935, 2004.

[6] L. Jin, K. Parthasarathy, T. Kuyel, "High-Performance ADC Linearity Test Using Low-Precision Signals in Non-Stationary Environments", International Test Conference, pp. 1182 - 1191, 2005.

[7] A. Roy, S. Sunter, A. Fudoli, "High Accuracy Stimulus Generation for A/D Converter BIST", International Test Conference, pp. 1031-1039, 2002.

[8] D. De Venuto, L. Reyneri, "Full Digital Optimized Testing and Calibration Technique for $\Sigma \Delta$ ADCs", International Symposium on Quality Electronic Design, pp. 519-526, 2007. 
[9] M. da Bloria Flores, M. Negreiros, L. Carro, "INL and DNL Estimation based on Noise ADC Test", Instrumentation and Measurement Technology Conference, pp.1350-1353, 2003.

[10] Xiaoqin Sheng, Hans Kerkhoff, Amir Zjajo, Guido Gronthoud, "Algorithms for ADC multi-site test with digital input stimuli", European Test symposium, 2009.

[11] Rudy van de Plassche, Integrated Analogue-to-Digital and Digital-toAnalogue Converters, Kluwer Academic Publishers, 1994. 